\title{
MARTINGALES OF STRONGLY MEASURABLE PETTIS INTEGRABLE FUNCTIONS
}

\author{
BY \\ J. J. UHL, JR.( $\left.{ }^{1}\right)$
}

\begin{abstract}
This paper deals with convergence theorems for martingales of strongly measurable Pettis integrable functions. First, a characterization of those martingales which converge in the Pettis norm is obtained. Then it is shown that a martingale which is convergent in the Pettis norm converges to its limit strongly in measure and, if the index set is the positive integers, it converges strongly almost everywhere to its limit. The second part of the paper deals with the strong measure and strong almost everywhere convergence of martingales which are not necessarily convergent in the Pettis norm. The resulting theorems here show that $L^{1}$-boundedness can be considerably relaxed to a weaker control condition on the martingale by the use of some facts on finitely additive vector measures.
\end{abstract}

Convergence of martingales of Bochner integrable functions has been the theme of many papers written over the last ten years. On the other hand, aside from the work of Métivier, martingales of weakly integrable functions have received comparatively little attention, possibly because of the apparent lack of a real structure theory for Pettis integrable functions. The purpose of this paper is to strike a course halfway between the two areas mentioned above by studying martingales of strongly measurable Pettis integrable functions. In the first section various preliminary results are gathered to establish the setting for the remainder of the paper. The second section characterizes martingales which converge in the Pettis norm. The existence of such a characterization is a somewhat surprising fact in itself since there are nonconvergent martingales which are Cauchy in the Pettis norm. The last section is devoted to the problem of strong pointwise convergence of martingales of Pettis integrable strongly measurable functions. The results of this section are general enough to include theorems of Doob, Krickeberg, Chatterji, and the author dealing with measure convergence and almost everywhere convergence of $L^{1}$-bounded martingales of Bochner integrable functions.

1. Preliminaries. Throughout this paper $(\Omega, \Sigma, \mu)$ is a fixed finite measure space; $\mathfrak{X}$ is a Banach space with continuous dual $\mathfrak{X}^{*}$. A function $f: \Omega \rightarrow \mathfrak{X}$ is strongly measurable if $f$ is the almost everywhere $[\mu]$ limit of measurable simple functions

Received by the editors July 6, 1971.

AMS 1970 subject classifications. Primary 60G45; Secondary 46G10, 46E40, $28 \mathrm{~A} 45$.

Key words and phrases. Martingale, vector valued functions, Pettis integral, Bochner integral, strong almost everywhere convergence, strong measure convergence, Cauchy nonconvergent martingales.

(1) The author gratefully acknowledges partial support by NSF GP 20431. 
of the form $\sum_{i=1}^{n} x_{i} \chi_{E_{i}}, x_{i} \in \mathfrak{X}, E_{i} \in \Sigma$. A strongly measurable function $f: \Omega \rightarrow \mathfrak{X}$ is called Pettis integrable if $x^{*} f \in L^{1}(\Omega, \Sigma, \mu)\left(=L^{1}(\mu)\right)$ for all $x^{*} \in \mathfrak{X}^{*}$ and if for each $E \in \Sigma$, there exists $x_{E} \in \mathfrak{X}$ satisfying the identity $x^{*}\left(x_{E}\right)=\int_{E} x^{*} f d \mu$ for all $x^{*} \in \mathfrak{X}^{*}$. In this case one writes $x_{E}=P-\int_{E} f d \mu$. If also $\|f\| \in L^{1}(\mu)$, then $f$ is Bochner integrable and for the purposes of this paper we shall write Bochner- $\int_{E} f d \mu$ $=P-\int_{E} f d \mu=\int_{E} f d \mu$, the unadorned integral symbol meaning Bochner or Lebesgue integration. If $f$ is strongly measurable and Pettis integrable, we define

$$
\|f\|_{P_{1}}=\sup _{\left\|x^{*}\right\| \leqq 1} \int_{\Omega}\left|x^{*} f\right| d \mu .
$$

After identification of functions which agree on all but possibly a set of $\mu$-measure zero, the collection of all $\mathfrak{X}$ valued strongly measurable functions becomes a normed linear space which is typically incomplete $[12$, p. 303]. This space will be denoted by $P_{1}(\mathfrak{X})$ or $P_{1}(\mu, \mathfrak{X})$ or $P_{1}(\Sigma, \mu, \mathfrak{X})$ depending on the context. Pettis has shown [12, p. 284, Corollary 2.5.1] that if $f \in P_{1}(\mathfrak{X})$, then

$$
\lim _{\mu(E) \rightarrow 0}\left\|f \chi_{E}\right\|_{P_{1}}=0 .
$$

From this it is easy to prove

Lemma 1.1. Let $B$ be a subfield of $\Sigma$ such that the $\sigma$-field generated by $B$ is $\Sigma$. Then the linear span of the set $\left\{x \chi_{E}: x \in \mathfrak{X}, E \in B\right\}$ is dense in $P_{1}(\mathfrak{X})$. (Here $\chi_{E}$ is the characteristic or indicator function of the set $E$.)

Definition 1.2. Let $f \in P_{1}(\Sigma, \mathfrak{X})$ and $B$ be a sub- $\sigma$-field of $\Sigma$. $g \in P_{1}(B, \mathfrak{X})$ is called the conditional expectation of $f$ relative to $B$ if $P-\int_{E} g d i=P-\int_{E} f d \mu$ for all $E \in B$. In this case one writes $E^{B}(f)=g$.

Some comments on this definition are in order. $B$ is a sub- $\sigma$-field of $\Sigma$ and $g \in P_{1}(B, \mathfrak{X})$, then from [12, p. 291, Theorem 4.3] it follows that $g \in P_{1}(\Sigma, \mathfrak{X})$. Hence when $f \in P_{1}(\Sigma, \mathfrak{X})$ and $E^{B}(f)$ is defined then $E^{B}(f) \in P_{1}(\Sigma, \mathfrak{X})$. Also since $E^{B}$ is a contraction on $L^{1}(\Sigma)$ it follows from the definition of $\|\cdot\|_{P_{1}}$ that $E^{B}$ is a linear contraction on its domain. Now, in view of Lemma $1.1, L^{1}(\Sigma, \mathfrak{X})$, the space of all Bochner integrable functions is dense in $P_{1}(\Sigma, \mathfrak{X})$. Since $E^{B}$ is defined on all of $L^{1}(\Sigma, \mathfrak{X}), E^{B}$ is densely defined. However, at the time of this writing, it is not known whether $E^{B}$ is defined on all of $P_{1}(\Sigma, \mathfrak{X})$.

Definition 1.3. Let $T$ be a directed set and $\left\{B_{t}, \tau \in T\right\}$ be an increasing net of sub- $\sigma$-fields of $\Sigma$; i.e. $\tau_{1} \leqq \tau_{2}$ implies $B_{\tau_{1}} \subset B_{\tau_{2}} \cdot\left\{f_{\imath}, B_{\imath}, \tau \in T\right\} \subset P_{1}(\Sigma, \mathfrak{X})$ is a martingale if $\tau_{1} \leqq \tau_{2}$ implies $E^{B_{\tau_{1}}}\left(f_{\tau_{2}}\right)=f_{\tau_{1}}$.

The next two lemmas are the keys to the work of $\S \S 2$ and 3.

LEMmA 1.4. For a martingale $\left\{f_{\imath}, B_{\imath}, \tau \in T\right\}$ in $P_{1}(\Sigma, \mathfrak{X})$, the following conditions are equivalent:

(i) $\lim _{\tau} f_{\tau}$ exists in $P_{1}$-norm.

(ii) There exists $f \in P_{1}\left(\sigma\left(\bigcup_{\imath} B_{\imath}\right), \mathfrak{X}\right)\left(\subset P_{1}(\Sigma, \mathfrak{X})\right)$ such that $E^{B_{\imath}}(f)=f_{\tau}$ for all $\tau \in T$. 
(iii) There exists $f \in P_{1}\left(\sigma\left(\bigcup_{\tau} B_{\tau}\right), \mathfrak{X}\right)$ such that $\lim _{\tau} P-\int_{E} f_{\tau} d \mu=P-\int_{E} f d \mu, E$ $\in \bigcup_{\tau} B_{\tau}$.

Proof. Since $P_{1}\left(\sigma\left(\bigcup_{\tau} B_{\tau}\right), \mathfrak{X}\right)$ is a closed subspace of $P_{1}(\Sigma, \mathfrak{X})$ we can and do assume that $\sigma\left(\bigcup_{\tau} B_{\tau}\right)=\Sigma$. To prove (i) $\Rightarrow$ (ii) and (iii), note that if $\lim _{\tau} f_{\tau}=f$ in $P_{1}$-norm, then since Pettis integration is a continuous operation, $\lim _{\tau} P-\int_{E} f_{\tau} d \mu$ $=P-\int_{E} f d \mu$, for all $E \in \bigcup_{\tau} B_{\tau}$. Hence (i) $\Rightarrow$ (iii). Also from the martingale property, $P-\int_{E} f_{\tau_{0}} d \mu=P-\int_{E} f d \mu$ for $E \in B_{\tau_{v}}$. Hence $E^{B_{\tau_{0}}}(f)=f_{\tau}$. Thus (i) $\Rightarrow$ (iii). To prove (iii) $\Rightarrow$ (ii) $\Rightarrow$ (i), note that the martingale property shows immediately that (iii) $\Rightarrow$ (ii). Now assume (ii) and let $\varepsilon>0$ be given. By Lemma 1.1 there exists $f_{\varepsilon}=\sum_{i=1}^{n} x_{i} \chi_{E_{i}}, E_{i} \in \bigcup_{\tau} B_{\tau}$ such that $\left\|f-f_{\varepsilon}\right\|_{P_{1}}<\varepsilon / 2$. Choosing $\tau_{0}$ such that $\left\{E_{i}\right\}_{i=1}^{n}$ $\subset B_{\tau_{0}}$ yields $E^{B_{\imath}}\left(f_{\varepsilon}\right)=f_{\varepsilon}$ for $\tau \geqq \tau_{0}$ and

$$
\begin{aligned}
\left\|f-f_{\tau}\right\|_{P_{1}} & \leqq\left\|f-f_{\varepsilon}\right\|_{P_{1}}+\left\|f_{\varepsilon}-f_{\tau}\right\|_{P_{1}} \\
& =\left\|f-f_{\varepsilon}\right\|_{P_{1}}+\left\|E^{B_{\tau}}\left(f_{\varepsilon}-f_{\tau}\right)\right\|_{P_{1}} \leqq 2\left\|f-f_{\varepsilon}\right\|_{P_{1}}<\varepsilon .
\end{aligned}
$$

Hence (ii) $\Rightarrow$ (i).

This section will close with an adaptation of the Radon-Nikodym theorem for the Pettis integral of [10] to a form suitable in the current context. The relationship between the next result and Lemma 1.4 will become clear in the next section if not sooner.

LEMMA 1.5. Let $\Sigma_{0}$ be a subfield of $\Sigma$ such that $\sigma\left(\Sigma_{0}\right)=\Sigma$. If $F$ is a countably additive measure $\mathfrak{X}$-valued vector measure defined on $\Sigma_{0}$, then $F$ admits the representation

$$
F(E)=P-\int_{E} f d \mu, \quad E \in \Sigma_{0},
$$

for some $f \in P_{1}(\Sigma, \mathfrak{X})$ if and only if

(i) $F$ has a bounded range.

(ii) $F$ is $\sigma$-continuous; i.e. $\lim _{\mu(E) \rightarrow 0}\|F(E)\|=0$.

(iii) Given $\varepsilon>0$ there exists a weakly compact convex subset $K \subset \mathfrak{X}$ such that for any $\delta>0$ there exists $E_{0} \in \Sigma_{0}$ with $\mu\left(\Omega-E_{0}\right)<\varepsilon$ and $F(E) \in \mu(E) K+\delta U$ for all $E \subset E_{0}, E \in \Sigma_{0}$, where $U$ is the closed unit ball of $\mathfrak{X}$.

Proof (Sufficiency). Let $F$ satisfy (i), (ii), and (iii). Since $F$ is $\mu$-continuous, $F$ has a $\mu$-continuous countably additive extension (still denoted by $F$ ) to all of $\Sigma$. Proceeding as in the proof of [16, Proposition 1, p. 274], there exists a set $S_{0} \in \Sigma$ such that $\mu\left(\Omega-S_{0}\right) \leqq \varepsilon$ and $F(E) \in \mu(E) K$ for all $E \subset S_{0}, E \in \Sigma$. An application of [10, Theorem 2] produces $f \in P_{1}(\Sigma, \mathfrak{X})$ such that $F(E)=P-\int_{E} f d \mu$ for all $E \in \Sigma$ and hence for all $E \in \Sigma_{0}$. This completes the proof of the sufficiency.

(Necessity). Suppose there exists $f \in P_{1}(\Sigma, \mathfrak{X})$ such that $F(E)=P-\int_{E} f d \mu$ for all $E \in \Sigma_{0}$ and let $\varepsilon>0$ be given. According to [10, Theorem 1], there exists $S_{0} \in \Sigma$, $\mu\left(\Omega-S_{0}\right)<\varepsilon / 2$ and a norm compact set $K$, which may be assumed convex by Mazur's theorem, such that $F(E) \in \mu(E) K$ for all $E \subset S_{0}, E \in \Sigma$. For a fixed $\delta>0$, 
proceeding as in the proof of [16, Proposition 1, p. 276], one can find $E_{0} \in \Sigma_{0}$, $\mu\left(\Omega-E_{0}\right)<\varepsilon$ such that $E \subset E_{0}, E \in \Sigma_{0}$ implies $F(E) \in \mu(E) K+\delta U$. This proves the necessity of (iii); since (i) and (ii) are clear, this completes the proof of the necessity.

A consequence of the proof of Lemma 1.5 is

COROLlARY 1.6. Lemma 1.5 remains true if the words "weakly compact" are replaced by "norm compact".

Another obvious corollary is

COROLlaRY 1.7. Lemma 1.5 remains true if $\mathfrak{X}$ is reflexive and the words "weakly compact" are replaced by "bounded".

2. Mean convergence in $P_{1}(\Sigma, \mathfrak{X})$. The goal of this section is to characterize martingales which converge in $P_{1}(\Sigma, \mathfrak{X})$-norm. In the more general case of locally convex spaces $\mathfrak{X}$, Métivier has given conditions under which a martingale $\left\{f_{\tau}, B_{\imath}, \tau \in T\right\}$ of weakly integrable functions converges weakly in the mean in the sense that there exists a weakly measurable $f$ such that $\lim _{\tau} \int_{\Omega}\left|x^{*}\left(f_{\tau}-f\right)\right| d \mu=0$ for all $x^{*} \in \mathfrak{X}^{*}$. In the case of Banach space valued strongly measurable functions, a considerably stronger result can be proved.

THEOREM 2.1. Let $\left\{f_{\tau}, B_{\tau}, \tau \in T\right\}$ be a martingale in $P_{1}(\mathfrak{X})$. The net $\left\{f_{\tau}, \tau \in T\right\}$ is norm convergent to some function $f \in P_{1}(\mathfrak{X})$ if and only if

(i) $\sup _{\tau}\left\|f_{\tau}\right\|_{P_{1}}<\infty$.

(ii) For each $\varepsilon>0$ there is $a \delta>0$ and index $\tau_{0} \in T$ such that $\mu(E)<\delta$ implies $\left\|f_{\tau} \chi_{E}\right\|_{P_{1}}<\varepsilon$ for all $\tau \geqq \tau_{0}$.

(iii) For each $\varepsilon>0$, there is a weakly compact convex set $K \subset \mathfrak{X}$ such that for any $\delta>0$ there is an index $\tau_{0}$ and a set $E_{0} \in B_{\tau_{0}}, \mu\left(\Omega-E_{0}\right)<\varepsilon$ such that $\tau \geqq \tau_{0}$ implies $P-\int_{E} f_{\tau} d \mu \in \mu(E) K+\delta U$ for all $E \subset E_{0}, E \in B_{\tau}$.

Proof. Again since $P\left(\sigma\left(\bigcup_{\tau} B_{\tau}\right), \mathfrak{X}\right)$ is a closed subspace of $P(\Sigma, \mathfrak{X})$, there is no loss of generality in assuming $\sigma\left(\bigcup_{\tau} B_{\tau}\right)=\Sigma$.

(Necessity). Suppose $\lim _{\tau}\left\|f_{\tau}-f\right\|_{P_{1}}=0$ for some $f \in P_{1}(\mathfrak{X})$. By Lemma 1.4, $E^{B_{\imath}}(f)=f_{\tau}$ for each $\tau \in T$. Also since $E^{B_{\tau}}$ is a contraction, $\left\|f_{\tau}\right\|_{P_{1}}=\left\|E^{B_{\tau}}(f)\right\|_{P_{1}}$ $\leqq\|f\|_{P_{1}}$. This proves (i). To prove (ii) note that

$$
\lim _{\tau}\left|\left\|f_{\tau} \chi_{E}\right\|_{P_{1}}-\left\|f \chi_{E}\right\|_{P_{1}}\right| \leqq \lim _{\tau}\left\|f_{\tau} \chi_{E}-f \chi_{E}\right\|_{P_{1}} \leqq \lim _{\tau}\left\|f_{\tau}-f\right\|_{P_{1}}=0
$$

uniformly in $E \in \Sigma$. (ii) now follows directly from the fact that $\lim _{\mu(E) \rightarrow 0}\left\|f \chi_{E}\right\|_{P_{1}}=0$. To prove (iii), let $\varepsilon>0$ be given. By Corollary 1.6, there is a norm compact convex set $K \subset \mathfrak{X}$ such that given $\delta>0$, there is $E_{\delta} \in \bigcup_{\tau} B_{\tau}$ such that

$$
P-\int_{E} f d \mu \in \mu(E) K+\delta U
$$

for $E \subset E_{\delta}, E \in \bigcup_{\tau} B_{\tau}$. For a fixed $\delta>0$, select $\tau_{0} \in T$ such that $E_{\delta} \in B_{\tau_{0}}$. Then for $\tau_{0} \leqq \tau$ and $E \in B_{\imath}, E \subset E_{\delta}$, from the martingale property one has 


$$
P-\int_{E} f_{\tau} d \mu=P-\int_{E} f d \mu \in \mu(E) K+\delta U .
$$

This proves the necessity.

(Sufficiency). Define the set function $F$ on $\bigcup_{\tau} B_{\tau}$ by

$$
F(E)=\lim _{\tau} \int_{E} f_{\imath} d \mu, \quad E \in \bigcup_{\tau} B_{\imath} .
$$

The martingale property ensures that the defining net on the right is eventually constant for $E \in \bigcup_{\tau} B_{\tau}$. Thus $F$ is well defined and obviously finitely additive on $\bigcup_{\tau} B_{\tau}$. Moreover

$$
\begin{aligned}
\|F(E)\| & =\sup _{\left\|x^{*}\right\| \leqq 1}\left|x^{*} F(E)\right| \\
& \leqq \sup _{\left\|x^{*}\right\| \leqq 1} \lim \sup _{\tau} \int_{E}\left|x^{*} f_{\tau}\right| d \mu \leqq \lim \sup _{\tau}\left\|f_{\tau} \chi_{E}\right\|_{P_{1}} .
\end{aligned}
$$

Hence (ii) of the hypothesis implies $\lim _{\mu(E) \rightarrow 0}\|F(E)\|=0$. Hence $F$ is $\mu$-continuous and is therefore countably additive on $\bigcup_{\tau} B_{\tau}$. A similar computation together with (i) shows that $F$ is of finite semivariation on $\bigcup_{\tau} B_{\tau}$. In view of Lemma 1.4 the proof will be complete if it can be shown that there exists $f \in P_{1}(\Sigma, \mathfrak{X})$ such that $\lim _{\tau} P-\int_{E} f_{\tau} d \mu=P-\int_{E} f d \mu$ for all $E \in \bigcup_{\tau} B_{\tau}$. For this, let $\varepsilon>0$ be given and let $K$ be as in (iii). By (iii) for any fixed choice of $\delta>0$, there exist $\tau_{0} \in T$ and $E_{0} \in B_{\tau_{0}}$, $\mu\left(\Omega-E_{0}\right)<\varepsilon$ such that

$$
P-\int_{E} f d \mu \in \mu(E) K+\delta U
$$

for $E \subset E_{0}$. Thus if $E \in \bigcup_{\tau} B_{\imath}, E \in \tau_{1}$ for some $\tau_{1} \geqq \tau_{0}$ and $F(E)=P-\int_{E} f_{\tau} d \mu \in \mu(E) K$ $+\delta U$. According to Lemma 1.5 there exists $f \in P_{1}(\Sigma, \mathfrak{X})$ such that $F(E)=P-\int_{E} f d \mu$, $E \in \bigcup_{\tau} B_{\tau}$. In view of Lemma 1.4, this completes the proof of the sufficiency.

The corollary below follows directly from the proof of the above theorem and Corollaries 1.6 and 1.7.

COROLlaRY 2.2. Theorem 2.1 remains true if the words "weakly compact" are replaced by "norm compact". If $\mathfrak{X}$ is reflexive, Theorem 2.1 remains true if the words "weakly compact" are replaced by "bounded".

Now let $\left\{f_{\tau}, B_{\tau}, \tau \in T\right\}$ be a norm convergent martingale in $P_{1}(\mathfrak{X})$ with limit $f$. Since $f$ is strongly measurable $\|f\|\left(=\|f\|_{x}\right)$ is measurable. Hence if $G_{n}=[\|f\| \leqq n]$, then $\left\{G_{n}\right\} \subset \sigma\left(\bigcup_{\tau} B_{\tau}\right)=\Sigma$ and $G_{n} \uparrow \Omega$. Therefore if $\lambda: \Sigma \rightarrow R$ is defined by $\lambda(E)$ $=\int_{E}\|f\| d \mu, \lambda$ is $\sigma$-finite. Consequently if $\varepsilon>0$ is given there exists $n_{0}$ such that if $E_{0}=G_{n_{0}}, \mu\left(\Omega-E_{0}\right)<\varepsilon / 2$ and $\lambda\left(E_{0}\right)<\infty$. Noting that $\mu$ and $\lambda$ can be reconstructed on $\Sigma=\sigma\left(\bigcup_{\tau} B_{\tau}\right)$ from their values on $\bigcup_{\tau} B_{\tau}$ by use of outer measures, one can see that there exists a disjoint sequence $\left\{S_{n}\right\} \subset \bigcup_{\tau} B_{\tau}$ such that $\bigcup_{n} S_{n} \supset E_{0}, \sum_{n=1}^{\infty} \lambda\left(S_{n}\right)$ $<\infty$, for, if there is no such sequence, then $\lambda\left(E_{0}\right)=+\infty$. Now select $m$ such that $\mu\left(\bigcup_{n \geqq m} S_{n}\right)<\varepsilon / 2$ and put $E_{1}=\bigcup_{n \leqq m} S_{n}$. Then $\lambda\left(E_{1}\right)<\infty$ and $\mu\left(\Omega-E_{1}\right)$ 
$=\mu\left(\Omega-\bigcup_{n} S_{n}\right)+\mu\left(\bigcup_{n} S_{n}-E_{1}\right) \leqq \mu\left(\Omega-E_{0}\right)+\mu\left(\bigcup_{n \geqq m} S_{n}\right)<\varepsilon / 2+\varepsilon / 2=\varepsilon$. Since $\left\{B_{\imath}\right.$, $\tau \in T\}$ is increasing, there exists $\tau_{1} \in T$ such that $E_{1} \in B_{\tau}$ for $\tau \geqq \tau_{1}$. Now consider the martingale $\left\{f_{\tau} \chi_{E_{1}}, B_{\tau}, \tau \in T, \tau \geqq \tau_{1}\right\}$ since $\lim _{\tau}\left\|f_{\tau}-f\right\|_{P_{1}}=0, E^{B_{\tau}}(f)=f_{\tau}$ for all $\tau$ and for $\tau \geqq \tau_{1}$. $E^{B_{\tau}}\left(f \chi_{E_{1}}\right)=E^{B_{\tau}}(f) \chi_{E_{1}}=f_{\tau} \chi_{E_{1}}$. But $\int_{\Omega}\left\|f \chi_{E_{1}}\right\| d \mu=\lambda\left(E_{1}\right)<\infty$. Hence $f \chi_{E_{1}} \in L^{1}(\mathfrak{X})$. It follows that $\lim _{\tau}\left\|f_{\tau} \chi_{E_{1}}-f \chi_{E_{1}}\right\|_{L^{1}}=0$. Hence $\lim _{\tau} f_{\tau} \chi_{E}=f \chi_{E_{1}}$ strongly in $\mu$-measure. Since $\mu\left(\Omega-E_{1}\right)<\varepsilon$ and $\varepsilon>0$ is arbitrary, it follows that $\lim _{\tau} f_{\tau}=f$ strongly in $\mu$-measure. This proves the first part of the following theorem:

THEOREM 2.3. Let $\left\{f_{\tau}, B_{\tau}, \tau \in T\right\}$ be a convergent martingale in $P_{1}(\mathfrak{X})$ with limit $f$. Then $\lim _{\tau} f_{\tau}=f$ strongly in $\mu$-measure. If $T=N$, the positive integers with natural order, then $\lim _{n} f_{n}=f$ strongly a.e. $[\mu]$.

Proof. Only the second assertion needs a proof. Returning to the end of the above argument one has $\lim _{n}\left\|f_{n} \chi_{E_{1}}-f_{\chi_{E}}\right\|_{L^{1}}=0$. An appeal to a theorem of $\mathrm{A}$. and C. Ionescu-Tulcea [7, p. 198] and Neveu [11, p. 32] shows that $\lim _{n} f_{n} \chi_{E_{1}}=f \chi_{E_{1}}$ strongly a.e. $[\mu]$. Again, since $\mu\left(\Omega-E_{1}\right)<\varepsilon$ and $\varepsilon>0$ is arbitrary, it follows that $\lim _{n} f_{n}=f$ strongly a.e. $[\mu]$.

For some Banach spaces $\mathfrak{X}$, for instance separable dual spaces and reflexive spaces, the basic theory of convergence of $L^{1}(\Sigma, \mathfrak{X})$ martingales is very similar to the theory of convergence of $L^{1}(\Sigma, R)$ martingales [3]. Unfortunately in the case of $P_{1}(\Sigma, \mathfrak{X})$ martingales there seems to be no nontrivial conditions one can place on $\mathfrak{X}$ to obtain a simple theory of convergence. For even when $\mathfrak{X}$ is a separable Hilbert space, trouble can occur.

Fact 2.4. If $\mathfrak{X}$ is a separable infinite dimensional Hilbert space and $\mu$ is Lebesgue measure, there is a nonconvergent norm Cauchy martingale in $P_{1}($ Borel sets, $\mathfrak{X})$.

Proof. The example is a small adaptation of an example originally due to $G$. Birkhoff and later used by Pettis for a similar purpose. Let $\left\{x_{i j}\right\}$ be an infinite orthonormal set in $\mathfrak{X}$. Set

$$
\begin{aligned}
y_{i}(s) & =x_{i j} & & \text { for } s \in\left[\left(j-1 / 2^{i}, 2 j-1 / 2^{i+1}\right)\right], \\
& =-x_{i j} & & \text { for } s \in\left[\left(2 j-1 / 2^{i+1}, j / 2^{i}\right)\right], \\
& =0 & & \text { for } s \in\{0,1\},
\end{aligned}
$$

for $j=1,2 \cdots 2^{i}$.

Put $f_{n}(s)=\sum_{i=1}^{n} y_{i}(s)$ for $s \in[0,1]$. Clearly $\left\{f_{n}\right\}$ is a martingale in $P_{1}$ (Borel sets, $\mathfrak{X ) . ~ M o r e o v e r ~}$

$$
\begin{aligned}
\left\|y_{i}\right\|_{P_{1}} & =\sup _{\left\|x^{*}\right\| \leqq 1} \int_{0}^{1}\left|x^{*} y_{i}\right| d \mu=\sup _{\left\|x^{*}\right\| \leqq 1} \sum_{j=1}^{2^{i}}\left|x^{*}\left(x_{i j}\right)\right| 2^{-i} \\
& \leqq 2^{-i} \sup _{\left\|x^{*}\right\| \leqq 1}\left(\sum_{j=1}^{2^{i}}\left|x^{*}\left(x_{i j}\right)\right|^{2}\right)^{\cdot / 2}\left(2^{i}\right)^{1 / 2}
\end{aligned}
$$

by the Cauchy-Bunyakowski-Schwarz inequality,

$$
\leqq 2^{-i}(1) 2^{i / 2}=2^{-i / 2} .
$$


Hence $\left\|f_{n}-f_{m}\right\|_{P_{1}} \leqq \sum_{i=m+1}^{n} 2^{-i / 2}$ and $\left\{f_{n}\right\}$ is Cauchy in $P_{1}$-norm.

Now suppose $\left\{f_{n}\right\}$ converges in $P_{1}$-norm to $f \in P_{1}$ (Borel sets, $\left.\mathfrak{X}\right)$. Then according to Theorem $2.3, \lim _{n} f_{n}=f$ strongly a.e. $[\mu]$. This is impossible since

$$
\lim _{n}\left\|f_{n}\right\|=+\infty \text { a.e. }[\mu] \text {. }
$$

This example serves to illuminate the role of (iii) of the hypothesis of Theorem 2.1. When $\mathfrak{X}$ is reflexive or is a separable dual space, (iii) of the hypothesis is satisfied for all $L^{1}$-bounded martingales [16, Theorem 5, p. 281]. The above example shows that the situation, even in the reflexive or separable dual case, is touchy enough to require the explicit presence of (iii) of the hypothesis of Theorem 2.1.

The next section extends Theorem 2.3 to a wider class of $P_{1}$-bounded martingales.

3. Strong measure and pointwise convergence. This section is devoted to studying strong pointwise and measure convergence of martingales in $P_{1}(\mathfrak{X})$. For the case of weak measure and pointwise convergence, this problem has been investigated by Métivier in [9]. For motivation, consider an $L^{1}(\mathfrak{X})$-bounded martingale $\left\{f_{n}, B_{n}, n \in N\right\}$. In [16, Theorem 4, p. 279], it was shown that if $\left\{f_{n}, B_{n}, n \in N\right\}$ satisfies (iii) of the hypothesis of Theorem 2.1 , then $\lim _{n} f_{n}$ exists strongly almost everywhere. Such a martingale has two properties worth noting: If $F(E)$ $=\lim _{n} \int_{E} f_{n} d \mu, E \in \bigcup_{n} B_{n}, F$ is continuous relative to some finitely additive finite measure on $\bigcup_{n} B_{n}$, namely its variation. Consequently [17, Corollary 3], $F\left(\bigcup_{n} B_{n}\right)$ is contained in some weakly compact set. This suggests that possibly $L^{1}(\mathfrak{X})$-boundedness can be relaxed to one of these conditions placed on $F$. The following theorem shows that this possibility is indeed a certainty.

THEOREM 3.1. Let $\left\{f_{\tau}, B_{\tau}, \tau \in T\right\}$ be a martingale in $P_{1}(\Sigma, \mathfrak{X})$ such that

(i) $\sup _{\tau}\left\|f_{\tau}\right\|_{P_{1}}<\infty$,

(ii) $\left\{P-\int_{E} f_{\tau} d \mu: E \in B_{\tau}, \tau \in T\right\}$ is contained in a weakly compact set $L \subset \mathfrak{X}$, and

(iii) for each $\varepsilon>0$ there is a weakly compact set $K \subset \mathfrak{X}$ such that for each $\delta>0$, there is an index $\tau_{0}$ and a set $E_{0} \in B_{\tau_{0}}, \mu\left(\Omega-E_{0}\right)<\varepsilon$ such that $\tau \geqq \tau_{0}$ implies $P-\int_{E} f_{\tau} d \mu \in \mu(E) K+\delta U$ for all $E \subset E_{0}, E \in B_{\tau}$.

Then the net $\left\{f_{\tau}, \tau \in T\right\}$ converges strongly in measure to $f \in P_{1}(\Sigma, \mathfrak{X})$. If $T=N$ with natural order, then $\lim _{n} f_{n}=f$ strongly a.e. $[\mu]$ as well.

Proof. Without loss of generality assume $\sigma\left(\bigcup_{\tau} B_{\tau}\right)=\Sigma$. Write $B=\bigcup_{\tau} B_{\tau}$. According to $[6, I V, 9.10-11]$ there exists a totally disconnected compact Hausdorff space $\Omega_{1}$ such that $B_{1}$, the field of all clopen subsets of $S_{1}$, is isomorphic to $B$ under a Boolean isomorphism $\alpha: B \rightarrow B_{1}$. Moreover every finitely additive real valued set function on $B_{1}$ is countably additive. If $\mu_{1}$ is defined on $B_{1}$ by $\mu_{1}(\alpha(E))=\mu(E)$, $E \in B$, and then extended to $\sigma\left(B_{1}\right)=\Sigma_{1}$, it is not difficult to establish the following facts. If $\varphi\left(\chi_{E}\right)=\chi_{\alpha(E)}$ for $E \in B$, then $\varphi$ has an extension as an isometry to $\operatorname{TM}(\Omega, \Sigma, \mu)=T M(\Sigma, \mu)$, the space of $\mu$-measurable scalar valued functions on $\Omega$ such that for $E \in \Sigma$, there exists $S \in \Sigma_{1}$ such that $\varphi\left(\chi_{E}\right)=\chi_{s}$ a.e. $\left[\mu_{1}\right]$ and if $S \in \Sigma_{1}$ 
there is $E \in \Sigma$ such that $\chi_{E}=\varphi^{-1}\left(\chi_{S}\right)$ a.e. $[\mu]$. Moreover $\varphi: T M(\mu) \rightarrow \operatorname{TM}\left(\mu_{1}\right)$ establishes an isometric isomorphism between the spaces indicated. Furthermore $\varphi \mid L^{1}(\mu)$ is onto $L^{1}\left(\mu_{1}\right)$ and establishes an isometric isomorphism between these spaces. Also since

$$
\|f\|_{\infty}=\sup \left\{\int_{E}|f| d \mu / \mu(E): E \in \Sigma, \mu(E)>0\right\}
$$

for $f \in L^{\infty}(\mu)$, it follows that $\varphi$ establishes an isometric isomorphism between $L^{\infty}(\mu)$ and $L^{\infty}\left(\mu_{1}\right)$. Finally if $\left\{f_{n}\right\} \subset T M(\mu)$ is such that $\lim _{n} f_{n}=f$ a.e. $\left[\mu_{1}\right]$, then for each $\varepsilon>0$, by Egoroff's theorem, there exists $E \in \Sigma, \mu(\Omega-E)<\varepsilon$ such that $\left\|\left(f_{n}-f\right) \chi_{E}\right\|_{\infty}$ $\rightarrow 0$. Hence $\left\|\left(\varphi\left(f_{n}\right)-\varphi(f)\right) \chi_{S}\right\|_{\infty} \rightarrow 0$ for $S \in \Sigma_{1}$ chosen such that $\varphi^{-1}\left(\chi_{S}\right)=\chi_{E}$ a.e. [ $\mu$ ]. Since $\mu_{1}(\Omega-S)<\varepsilon$, it follows that $\varphi\left(f_{n}\right) \rightarrow \varphi(f)$ a.e. [ $\left.\mu_{1}\right]$. Similarly if $\left\{\varphi\left(f_{n}\right)\right\} \subset T M\left(\mu_{1}\right)$ is such that $\varphi\left(f_{n}\right) \rightarrow \varphi(f)$ a.e. $\left[\mu_{1}\right]$, then $f_{n} \rightarrow f$ a.e. $[\mu]$. The same line of reasoning can be extended in view of [12, Theorem 4.3, p. 291] to show $P_{1}(\mu, \mathfrak{X})$ and $P_{1}\left(\mu_{1}, \mathfrak{X}\right)$ are isometrically isomorphic under an isometric isomorphism $\theta: f \rightarrow \bar{f}$ from $P_{1}(\mu, \mathfrak{X})$ onto $P_{1}\left(\mu_{1}, \mathfrak{X}\right)$ which satisfies

$$
P-\int_{E} f d \mu=P-\int_{\alpha(E)} \bar{f} d \mu \quad \text { for } E \in B .
$$

Accordingly, if $\left\{f_{\tau}, B_{\tau}, \tau \in T\right\}$ is a martingale in $P_{1}(\mu, \mathfrak{X})$, then $\left\{\bar{f}_{\tau}, \bar{B}_{\tau}, \tau \in T\right\}$, where $\bar{B}_{\tau}=\sigma\left(\alpha\left(B_{\tau}\right)\right)$ is a martingale in $P_{1}\left(\mu_{1}, \mathfrak{X}\right)$.

Now suppose $\left\{f_{\tau}, B_{\imath}, \tau \in T\right\}$ is a martingale in $P_{1}(\mu, \mathfrak{X})$ and satisfies the hypothesis of the theorem. Set $F(E)=\lim _{\tau} P-\int_{E} f_{\tau} d \mu$ for $E \in B$. $F$ is finitely additive and condition (ii) shows that $F(B)$ is contained in a weakly compact set. An application of [17, Corollary 3] establishes the existence of a finite nonnegative finitely additive measure $v$ on $B$ such that $\lim _{v(E) \rightarrow 0}\|F(E)\|=0$. Define $v_{1}$ on $B_{1}$ by $v_{1}(\alpha(E))$ $=v(E)$ for $E \in B$. $v_{1}$ is countably additive and hence has a countably additive extension, still denoted by $v_{1}$, to all of $\Sigma_{1}$. Similarly define $F_{1}$ on $B_{1}$ by $F_{1}(\alpha(E))$ $=F(E)$ for $E \in B$. Obviously $F_{1}$ is $v_{1}$-continuous on $B_{1}$. Now invoke condition (iii). From (iii) it follows that for each $\varepsilon>0$ there is a weakly compact set $K \subset \mathfrak{X}$ such that if $\delta>0$ is given, there is a set $E_{0} \in B, \mu\left(\Omega-E_{0}\right)<\varepsilon$ such that $F(E)$ $\in \mu(E) K+\delta U$ for all $E \subset E_{0}, E \in B$. Hence $F_{1}(S) \in \mu_{1}(S) K+\delta U$ for all $S \subset \alpha\left(E_{0}\right)$, $S \in B_{1}$ and $\mu_{1}\left(\Omega_{1}-\alpha\left(E_{0}\right)\right)<\varepsilon$. At this point, write $\lambda_{1}=\mu_{1}+v_{1}$. For $S \in B_{1}$, one has $\mu_{1}(S)=\left(\lambda_{1}(S)\right)\left(\mu_{1}(S) / \lambda_{1}(S)\right)$ if $\lambda_{1}(S) \neq 0$. Hence for $S \subset \alpha\left(E_{0}\right), S \in B, F_{1}(S) \in \lambda_{1}(S) K_{1}$ $+\delta U$ where $K_{1}=\{\beta x: 0 \leqq \beta \leqq 1, x \in K\}$. Obviously $K_{1}$ is weakly compact. Since $K_{1}$ is weakly compact, Lemma 1.5 applies and provides a function $g \in P_{1}\left(\lambda_{1}, \mathfrak{X}\right)$ such that $F_{1}(S)=P-\int_{S} g d \lambda_{1}$ for $S \in B_{1}$. Now let $h=d \mu_{1} / d \lambda_{1}$ and $h_{\tau}=E^{\bar{B}_{\tau}}(h)$. Then $\left\{h_{\tau}, B_{\imath}, \tau \in T\right\}$ is a convergent martingale in $L^{1}\left(\lambda_{1}\right)$ with limit $h$. Moreover $P-\int_{S} \bar{f}_{\tau} d \mu_{1}=P-\int_{S} \bar{f}_{\tau} h d \lambda_{1}=P-\int_{S} \bar{f}_{\tau} h_{\imath} d \lambda_{1}$ for all $S \in \sigma\left(B_{\tau}\right)$ for each fixed $\tau \in T$. By the martingale property $P-\int_{S} \bar{f}_{\tau} h_{\imath} d \lambda_{1}=F_{1}(S)=P-\int_{S} g d \lambda_{1}$ for $S \in \alpha\left(B_{\tau}\right)$. Hence $P-\int_{S} \bar{f}_{\tau} h_{\tau} d \lambda_{1}=P-\int_{S} g d \lambda$ for all $S \in \sigma\left(\alpha\left(B_{\tau}\right)\right)=\bar{B}_{\imath}$ for each fixed $\tau \in T$. Accordingly

$$
\lim _{\tau} P-\int_{S} \bar{f}_{\tau} h_{\imath} d \lambda_{1}=P-\int_{S} g d \lambda_{1}
$$


for all $S \in \bigcup_{\tau} \bar{B}_{\tau}$. By Lemma $1.4, \lim _{\tau} \bar{f}_{\tau} h_{\tau}=g$ in $P_{1}\left(\lambda_{1}, \mathfrak{X}\right)$. An appeal to Theorem 2.3 shows that $\lim _{\tau} \bar{f}_{\tau} h_{\tau}=g$ strongly in $\lambda_{1}$-measure. Hence $\lim _{\tau} \bar{f}_{\tau} h_{\tau}=g$ strongly in $\mu_{1}$-measure. Now $\mu_{1}[h=0]=0$ since $h=d \mu_{1} / d \lambda_{1}$. Thus $\lim _{\tau} \bar{f}_{\tau} h_{\tau} / h_{1}=g / h$ strongly in $\mu_{1}$-measure. But since $E^{\bar{B}_{\tau}}(h)=h_{\tau}, \lim _{\tau} h_{\tau}=h$ in $\lambda_{1}$-measure and hence in $\mu_{1}$-measure; i.e., $\lim _{\tau} h_{\tau} / h=1$ in $\mu_{1}$-measure. It follows that $\lim _{\tau} \bar{f}_{\tau}=g / h$ strongly in $\mu_{1}$-measure. From the remarks at the beginning of the proof, one finds that $\left\{f_{\tau}, \tau \in T\right\}$ is strongly Cauchy in $\mu$-measure.

Now examine $g / h$ again. Put $S_{0}=[h \neq 0]$. Then $\mu_{1}\left(\Omega_{1}-S_{0}\right)=0$ and

$$
\lim _{\mu_{1}(S) \rightarrow 0} \lambda_{1}\left(S \cap S_{0}\right)=0 .
$$

Put $\tilde{\lambda}_{1}(S)=\lambda_{1}\left(S \cap S_{0}\right), S \in \sigma\left(B_{1}\right)$. Then $d \tilde{\lambda}_{1} / d \mu_{1}=1 / h$. Hence $P-\int_{S} g d \lambda_{1}=\int_{S} g / h d \mu_{1}$ for $S \subset S_{0}$. Hence $g / h \in P_{1}\left(\mu_{1}, \mathfrak{X}\right)$. Choose $z \in P_{1}(\mu, \mathfrak{X})$ such that $P-\int_{E} z d \mu$ $=P-\int_{\alpha(E)} g / h d \mu_{1}$, for $E \in \bigcup_{\tau} B_{\tau}$. Then the fact that $\lim _{\tau} \bar{f}_{\tau}=g / h$ strongly in $\mu_{1}$ measure implies $\lim _{\tau} f_{\tau}=z$ strongly in $\mu$-measure.

If $T=N$, the proof that $\lim _{n} f_{n}=z \in P_{1}(\mu, \mathfrak{X})$ strongly a.e. [ $\left.\mu\right]$ is the same as the above if measure convergence is replaced by almost everywhere convergence.

Theorem 3.1 and its proof have three easy corollaries.

COROLLARY 3.2. In the statement of Theorem 3.1, (ii) may be replaced by

(ii)' There exists a finite nonnegative real valued finitely additive measure $v$ on $\bigcup_{\tau} B_{\tau}$ with the property that for each $\varepsilon>0$ there exists a $\delta>0$ such that $\left\|P-\int_{E} f_{\tau} d \mu\right\|<\varepsilon$ whenever $E \in B_{\tau}$ and $v(E)<\delta$.

Proof. The only use of (ii) in the proof of Theorem 3.1 was to guarantee the existence of such a measure.

COROLlary 3.3. If $\mathfrak{X}$ is reflexive, then in the statement of Theorem 3.1, (ii) may be dropped $\left({ }^{2}\right)$.

Proof. Bounded sets in reflexive Banach spaces are contained in weakly compact sets.

COROLlaRY 3.4 [16]. If $\left\{f_{\tau}, B_{\tau}, \tau \in T\right\}$ is an $L^{1}(\mu, \mathfrak{X})$-bounded martingale satisfying (iii) of the hypothesis of Theorem 3.1, then $\left\{f_{\tau}, B_{\tau}, \tau \in T\right\}$ converges strongly in measure to $f \in L^{1}(\mu, \mathfrak{X})$. If the index set $T=N$, then $\lim _{n} f_{n}=f$ strongly a.e. [ $\left.\mu\right]$ as well. If $\mathfrak{X}$ has the Radon-Nikodym property with respect to $\mu$, (iii) may be dropped.

Proof. To prove all but the last statement, note that an $L^{1}$-bounded martingale is $P_{1}$-bounded and that if $v(E)=\lim _{\tau} \int_{E}\left\|f_{\tau}\right\| d \mu$ for $E \in \bigcup_{\tau} B_{\tau}, v$ satisfies (ii)' of Corollary 3.2. The last statement is a consequence of [16, Theorem 5, p. 281] which says that if $\mathfrak{X}$ has the Radon-Nikodym property, (iii) is automatically satisfied for $L^{1}$-bounded martingales.

As a consequence of the last corollary, one sees that most of the classical and

$\left(^{2}\right)$ Professor J. Diestel has remarked that if $\mathfrak{X}$ contains no copy of $c_{0}$, then (ii) may be dropped. 
recent theorems ([2], [3], [7], [14], [15] and [16]) on the measure and almost everywhere convergence of $L^{1}(\mathfrak{X})$-bounded martingales are subsumed by the results of this section. It should be noted, that even the case that $\mathfrak{X}=$ the scalars, Theorem 3.1 yields a new proof of the classical almost everywhere convergence theorem of Doob.

\section{REFERENCES}

1. R. G. Bartle, N. Dunford and J. Schwartz, Weak compactness and vector measures, Canad. J. Math. 7 (1955), 289-305. MR 16, 1123.

2. S. D. Chatterji, Martingales of Banach-valued random variables, Bull. Amer. Math. Soc. 66 (1960), 395-398. MR 22 \#10008.

3. - Martingale convergence and the Radon-Nikodým theorem in Banach spaces, Math. Scand. 22 (1968), 21-41. MR 39 \#7645.

4. N. Dinculeanu, Vector measures, Internat. Series of Monographs in Pure and Appl. Math., vol. 95, Pergamon Press, Oxford; VEB Deutscher Verlag, Berlin, 1967. MR 34 \#6011b.

5. N. Dunford and B. J. Pettis, Linear operations on summable functions, Trans. Amer. Math. Soc. 47 (1940), 323-392. MR 1, 338.

6. N. Dunford and J. T. Schwartz, Linear operators. I: General theory, Pure and Appl. Math., vol. 7, Interscience, New York, 1958. MR 22 \#3302.

7. A. Ionescu-Tulcea and C. Ionescu-Tulcea, Abstract ergodic theorems, Trans. Amer. Math. Soc. 107 (1963), 107-124. MR 27 \#606.

8. I. Kluvánek, On the theory of vector measures. II, Mat.-Fyz. Casopis Sloven. Akad. Vied 16 (1966), 76-81. (Russian) MR 34 \#1475.

9. M. Métivier, Martingales à valeurs vectorielles. Applications à la dérivations des mesures vectorielles, Ann. Inst. Fourier (Grenoble) 17 (1967), fasc. 2, 175-208. MR 40 \#926.

10. S. Moedomo and J. J. Uhl, Jr., Radon-Nikodým theorems for the Bochner and Pettis integrals, Pacific J. Math. 38 (1971), 531-536.

11. J. Neveu, Relations entre les théories des martingales et la théorie ergodique, Colloq. Internat. Théorie du Potentiel, Paris, Juin 1964.

12. B. J. Pettis, On integration in vector spaces, Trans. Amer. Math. Soc. 44 (1938), 277-304.

13. R. S. Phillips, On weakly compact subsets of a Banach space, Amer. J. Math. 65 (1943), 108-136. MR 4, 218.

14. Ulf Rønnow, On integral representation of vector valued measures, Math. Scand. 21 (1967), 45-53. MR 39 \#4354.

15. F. S. Scalora, Abstract martingale convergence theorems, Pacific J. Math. 11 (1961), 347-374. MR 23 \#A684.

16. J. J. Uhl, Jr., Applications of Radon-Nikodým theorems to martingale convergence, Trans. Amer. Math. Soc. 145 (1969), 271-285. MR 40 \#4983.

17. - Extensions and decompositions of vector measures, J. London Math. Soc. 3 (1971), 672-676.

Department of Mathematics, University of Illinois, Urbana, Illinois 61801 